Orange Journal / Volumen 3 Número 5/ Enero - junio 2021

DOI: https://doi.org/10.46502/issn.2710-995X/2021.5.05

Carralero-Paredes, A., Suárez-León, A.A., Sóñora-Mengana, A., García-Naranjo, J.C. (2021). Detección de arritmias a partir de la determinación de la frecuencia cardiaca con fotopletismografía. Orange Journal, 3(5), 42-52. https://doi.org/10.46502/issn.2710-995X/2021.5.05

\title{
Detección de arritmias a partir de la determinación de la frecuencia cardiaca con fotopletismografía
}

\section{Arrhythmia detection based on heart rate from photoplethysmography}

Recibido: 23 de junio de 2021
Aceptado: 1 de agosto de 2021

Escrito por:

Arianna Carralero-Paredes ${ }^{15}$ https://orcid.org/0000-0003-1169-2375

Alexander A. Suárez-León ${ }^{16}$ https://orcid.org/0000-0002-9393-4552

Alexander Sóñora-Mengana ${ }^{17}$

https://orcid.org/0000-0003-1649-3383

Juan C. García-Naranjo ${ }^{18}$

https://orcid.org/0000-0001-7872-6758

\section{Resumen}

Las enfermedades cardiovasculares (ECV) cobran la vida de cerca de 18 millones de personas cada año, constituyendo la principal causa de muerte e incapacidad en el mundo. Entre las enfermedades cardiovasculares, las arritmias cardiacas son las más comunes. Desde hace varios años, nuevos estudios han destacado las potencialidades de la onda fotopletismográfica para detectar arritmias, superando en sencillez y reducción de costos a la electrocardiografía (ECG). En este estudio se propone un método de detección de picos sistólicos de la onda fotopletismográfica para determinar la frecuencia cardiaca y con ello establecer la presencia de taquicardia, bradicardia o asístole. El método de detección de picos sistólicos calcula la primera derivada de la señal previamente filtrada. A continuación aplica un proceso de umbralización. Finalmente, en una etapa de agrupamiento se emplea el algoritmo DBSCAN. El algoritmo de detección de picos fue evaluado en 42 señales de una base de datos internacional multiparamétrica para la estimación del RR. La evaluación del método mostró alta exactitud y precisión $(0 \pm 2 \mathrm{~ms})$ y una sensibilidad y valor predictivo positivo del $99 \%$. Estos resultados permiten determinar la frecuencia cardiaca con una exactitud y precisión de $0 \pm 1$ latido por minuto. Además, este algoritmo es evaluado en clasificación de arritmias utilizando 155 señales de la base de datos del PhysioNet/Computing in Cardiology Challenge del 2015. Para esta evaluación el algoritmo mostró resultados aceptables en la detección de asístole, bradicardia y taquicardia. La sensibilidad y el valor predictivo positivo fue del $79 \%$ y $88 \%$ para asístole, $74 \%$ y $64 \%$ para bradicardia y, $80 \%$ y $99 \%$ para taquicardia respectivamente. La efectividad del método puede afectarse en registros de señales con grandes variaciones de amplitud y/o con relaciones señal-ruido (SNR) bajas. No obstante, los resultados en estas condiciones son aceptables y son muy buenos en señales de alto SNR.

Palabras claves: arritmias, clasificación, detección de picos sistólicos, frecuencia cardiaca, onda fotopletismográfica.

\footnotetext{
${ }^{15}$ Ingeniera Biomédica. Centro Provincial de Electromedicina. Santiago de Cuba. Cuba.

${ }^{16}$ Dr. C Profesor Titular. Universidad de Oriente. Santiago de Cuba. Cuba.

${ }^{17}$ Dr. C. Investigador Agregado. Centro de biofísica Médica. Santiago de Cuba. Cuba.

${ }^{18}$ Dr. C Profesor Titular. Centro de biofísica Médica. Santiago de Cuba. Cuba.
} 


\begin{abstract}
Cardiovascular diseases (CVD) kill about 18 million people each year which constitute the leading cause of death and disability worldwide. Among cardiovascular diseases, cardiac arrhythmias are the most common. For several years, new studies have highlighted the potential of the photoplethysmographic wave to detect arrhythmias, surpassing in simplicity and cost reduction to electrocardiography (ECG). This study proposes a method of detecting systolic peaks of the photoplethysmographic wave to determine the heart rate and establish the presence of tachycardia, bradycardia, or asystole. The systolic peak detection method calculates the first derivative of the previously filtered signal. It then applies a thresholding process. Finally, in a clustering stage, the DBSCAN algorithm is used. The peak detection algorithm was evaluated on 42 signals from an international multiparametric database for RR estimation. The evaluation of the method showed high accuracy and precision $(0 \pm 2 \mathrm{~ms})$. The sensitivity and positive predictive value were $99 \%$. These results allow determining the heart rate with accuracy and precision of $0 \pm 1$ beats per minute. The algorithm was evaluated in arrhythmia classification using 155 signals from the PhysioNet/Computing in Cardiology Challenge 2015 database. For this evaluation, the algorithm showed acceptable results for detecting asystole, bradycardia, and tachycardia. The sensitivity and positive predictive values were $79 \%$ and $88 \%$ for asystole, $74 \%$ and $64 \%$ for bradycardia, and $80 \%$ and $99 \%$ for tachycardia, respectively. The method's effectiveness may be affected in signals with significant variations in amplitude or low signal-tonoise ratios (SNR). However, the results under these conditions are still acceptable and are very good at high SNR signals.
\end{abstract}

Key words: arrhythmias, classification, systolic peak detection, heart rate, photoplethysmographic wave.

\title{
Introducción
}

Las enfermedades cardiovasculares (ECV) son la principal causa de muerte a nivel mundial cobrando cerca de 17,9 millones de vidas cada año, lo cual representa un estimado del $32 \%$ de todas las muertes registradas en el mundo. Más de tres cuartas partes de las defunciones por ECV se producen en los países de ingresos bajos y medios (Coviello, 2020). En Cuba en el año 2020, las enfermedades cardiacas cobraron la vida de cerca de 30 mil personas, constituyendo la principal causa de muerte en el país. Entre las enfermedades cardiacas, las arritmias cardiacas son muy comunes. Las arritmias fueron responsables de 921 muertes en ese año (Constantén et al, 2020).

En la actualidad el método principal para diagnosticar arritmias es mediante la utilización del electrocardiograma (ECG). El ECG es la representación gráfica de la actividad eléctrica del corazón. Esta técnica es utilizada para valorar la condición del corazón en forma no invasiva. Pese a que el ECG es el estándar para la detección de arritmias, requiere de electrodos adheridos a la piel. Estos electrodos pueden causar irritación cutánea y necesitan ser reemplazados en intervalos regulares cuando se emplean en monitorización ambulatoria.

Desde hace varios años las señales fotopletismográficas se han convertido en un nuevo método para monitorizar el ritmo cardiaco. La técnica fotopletismográfica permite emplear dispositivos menos complejos y con menor número de electrodos que el ECG. La fotopletismografía (FPG) es una técnica de medición óptica simple y de bajo costo que puede emplearse para detectar los cambios de volumen sanguíneo en el lecho microvascular de un tejido (Castaneda et al, 2018) Las variaciones del volumen de sangre están en correspondencia con cada eyección del corazón, por lo que es posible determinar la frecuencia cardiaca midiendo las variaciones de la intensidad de la luz captada. La frecuencia cardiaca (FC) es numéricamente igual al inverso del intervalo entre despolarizaciones auriculares contiguas (Vázquez Seisdedos, 2001). La estimación de este parámetro permite detectar y analizar algunas arritmias cardiacas. Se denomina arritmia cardiaca a aquellos trastornos en la formación y/o conducción de los impulsos cardiacos que implican además una alteración del ritmo cardiaco (García et al, 2015). El rango normal de FC en la mayoría de los adultos es de 60-100 latidos por minuto (lpm) y varía en dependencia de la condición física de la persona. Una FC más elevada de lo normal se considera que la persona sufre de taquicardia; por el contrario, si la FC es más baja se considera bradicardia, mientras que la ausencia del ritmo cardiaco por más de 4 segundos se considera asístole (Saquib et al, 2015). 
Considerando lo anteriormente expuesto el objetivo de este trabajo es presentar un nuevo método de detección de picos sistólicos de la onda fotopletismográfica que permita determinar la frecuencia cardiaca y con ello detectar la presencia de taquicardia, bradicardia o asístole. Para ello se realizó primeramente un preprocesamiento a la señal y se detectaron los picos sistólicos empleando la fusión de dos métodos: derivada de la señal y un método de agrupamiento. Con los picos detectados se estimó la frecuencia cardiaca para finalmente discriminar entre cuatro grupos: normal, asístole, bradicardia y taquicardia.

\section{Marco Teórico}

La onda fotopletismográfica está formada por una componente pulsátil atribuida a los cambios cardiacos sincrónicos en el volumen de sangre con cada latido del corazón. Esta componente pulsátil está superpuesta en una línea base de variación lenta atribuidas a la respiración, la actividad del sistema nervioso simpático y la termorregulación (Allen, 2007). La sencillez de esta técnica hace que la FPG sea práctica para el monitoreo en el hogar y una buena alternativa para el diagnóstico de ECV. Esto ha motivado el interés en las investigaciones que utilizan las potencialidades de la FPG para detectar aquellas arritmias caracterizadas por alterar el ritmo cardiaco (Asensi et al, 2021).

Existen varios estudios para la detección y/o clasificación de arritmias. La mayoría de los estudios que utilizan solamente la onda fotopletismográfica están orientados a la identificación de fibrilación auricular (Bonomi et al, 2018), (Poh et al, 2018), (Rezaei Yousefi, 2018), (Shashikumar et al, 2017), (Stankevičius et al, 2016). Para otros tipos de arritmias además de la utilización tradicional del ECG se incorpora la onda fotopletismográfica (Eerikäinen et al, 2015), (Fallet et al, 2015), (Kalidas \& Tamil, 2015), (Clifford et al, 2015), (Sadr et al, 2015). No obstante, el uso de la FPG en la mayoría de los casos ha sido para mejorar el rendimiento de los algoritmos propuestos y no como método de detección o clasificación independiente. En (Paradkar \& Chowdhury, 2017) se propone un método que potencia el valor predictivo positivo en la detección de 4 tipos de ritmos irregulares: taquicardia, bradicardia, taquicardia ventricular y fibrilación ventricular. En (Polanía et al, 2015) se propone un método que utiliza Máquinas de Vectores de Soporte para clasificar usando fotopletismografía contracciones ventriculares prematuras (CVP) y taquicardia ventricular (TV) dentro de un grupo de señales que contienen además ritmo sinusal normal (RSN) y contracciones supraventriculares prematuras (CPSV). El presente estudio propone un algoritmo para detectar asístole, bradicardia y taquicardia, basado en la determinación de la FC a partir de la señal de FPG.

El artículo está estructurado como sigue: la sección Materiales y Métodos describe el algoritmo y la metodología de evaluación y en las secciones de Resultados y Discusión se muestran y discuten los resultados obtenidos. Finalmente se enuncian las conclusiones fundamentales.

\section{Materiales y Métodos}

Para la determinación de la frecuencia cardiaca a partir de la señal fotopletismográfica es necesario primeramente detectar los picos sistólicos. El método de detección de picos propuesto está compuesto por tres etapas de procesamiento: filtrado, búsqueda de candidatos y detección del pico sistólico, ver figura 1.

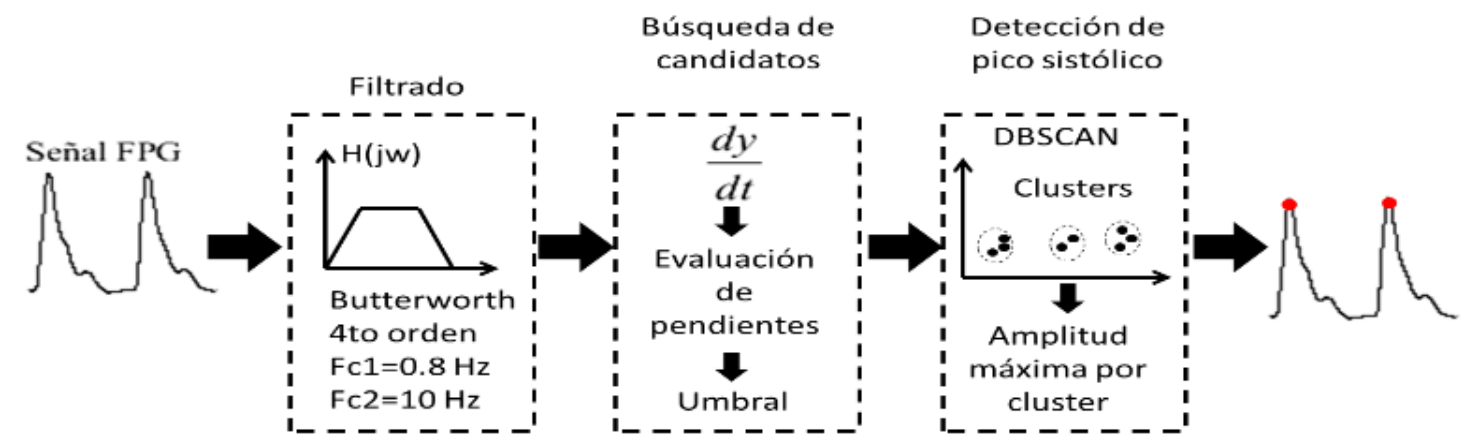

Figura 1. Diagrama en bloques del método de detección automática del pico sistólico. Elaboración propia. 


\section{ORANGE JOURNAL}

La FPG suele estar contaminada por ruidos y artefactos producto de: movimiento, variaciones de la luz ambiental, ubicación del sensor, respiración del paciente, características de la piel (humedad, color, grosor), entre otros (Jung et al., 2007). Para reducir la influencia de estos factores, el algoritmo incluye un filtro Butterworth pasa-banda de cuarto orden con $\mathrm{fc} 1=0.8 \mathrm{~Hz}$ y fc $2=10 \mathrm{~Hz}$ y fase cero (se trabajará con señales ya previamente adquiridas y anotadas).

El algoritmo opera en una ventana deslizante de 10 s con solapamiento del $25 \%$. En la ventana de análisis se determinan los máximos mediante la primera derivada haciendo una búsqueda de los cambios de signo de positivo a negativo. Para cada máximo se almacena el valor de la posición. Posteriormente se calculan dos umbrales, uno equivalente al $10 \%$ de la mediana de la señal y el otro equivalente al $30 \%$ de la amplitud máxima de la señal en la ventana de análisis. El primer umbral se toma para eliminar las pequeñas oscilaciones que pueden surgir alrededor de la línea base producto a la desconexión de los electrodos o a la ocurrencia de una asístole. El segundo umbral se utiliza para limitar la búsqueda de los candidatos, evitando la detección de varios picos diastólicos. Para cada ventana de análisis los valores de la FPG correspondientes a los máximos detectados en la derivada son comparados con los umbrales. Se almacena un 1 en la posición de cada pico que supera ambos umbrales y 0 en otro caso. El vector binario generado contiene las posiciones de los candidatos a picos sistólicos.

A continuación, el algoritmo DBSCAN (Density-Based Spatial Clustering of Applications with Noise) realiza un proceso de corrección de los picos detectados. DBSCAN es un algoritmo de agrupamiento basado en densidades diseñado para encontrar grupos de forma arbitraria (Ester et al, 1996). Este algoritmo se fundamenta en la idea de que los objetos que conforman una región densa (determinada por un umbral) deben agruparse juntos en un mismo grupo. El algoritmo busca regiones de alta densidad en el espacio de características que se encuentran separadas por regiones de menor densidad. DBSCAN agrupa los puntos que se encuentran a una distancia límite, que en este caso se ha tomado igual a la mitad del periodo refractario del corazón en muestras, 0.25 segundos (Guyton, 2006). En los parámetros de configuración de DBSCAN se emplea la función distancia 'cityblock'. Una vez realizado el agrupamiento se detecta la amplitud máxima de la señal correspondiente a cada uno de los grupos formados y se almacena la posición temporal de la misma.

El algoritmo de detección de picos se evaluó con la base de datos multiparamétrica CapnoBase.org para la estimación del RR (Karlen et al, 2013). Esta base de datos cuenta con 42 señales de electrocardiografía, fotopletismografía y capnografía muestreadas a $300 \mathrm{~Hz}$. Para la evaluación del algoritmo de detección de los picos sistólicos propuesto se determinó para cada registro la exactitud $(\mu)$ y la precisión $(\sigma)$ según,

$$
\mu=\frac{1}{N} \sum_{i=0}^{N} P_{r e f}-P_{\text {alg }} \quad \sigma=\sqrt{\frac{1}{N-1}\left(\left(P_{r e f}-P_{\text {alg }}\right)-\mu\right)^{2}}
$$

donde $N$ es el total de picos anotados en la base de datos.

Para evaluar la capacidad de detección del algoritmo se calculó para cada señal la sensibilidad ( $S e$ ), valor predictivo positivo $(\mathrm{P}+)$ y especificidad $(S p)$ mediante,

$$
S e=\frac{V P}{V P+F N} \quad \mathrm{P}+=\frac{V P}{V P+F P} \quad S p=\frac{V N}{V N+F P}
$$

Donde: VP -Verdadero Positivo, FN -Falso Negativo, FP -Falso Positivo

Como índice de rendimiento global se calcularon los valores medios de la exactitud y de la precisión en base a todos los registros. Una vez detectados los picos sistólicos se determinó la frecuencia cardiaca para cada señal utilizando la siguiente fórmula:

$$
\mathrm{FC}=(\text { No. Picos } \cdot \text { fs } \cdot 60) / \mathrm{n},
$$

siendo $n$ la muestra del último pico detectado y fs la frecuencia de muestreo. 
La clasificación de arritmias se realiza usando un subconjunto de la base de datos del PhysioNet/Computing in Cardiology Challenge 2015 (Clifford et al, 2015), (Eerikäinen et al, 2015). Esta base de datos incluye 750 registros de $5 \mathrm{~min}$ con eventos verdaderos y falsos de arritmias (asístole, bradicardia, taquicardia, fibrilación ventricular y taquicardia ventricular). Cada registro contiene dos derivaciones de ECG y una o más señales pulsátiles (fotopletismografía, presión de sangre arterial, respiración). Las señales han sido muestreadas a $250 \mathrm{~Hz}$ y recogen la anotación, ya sea verdadera o falsa, del tipo de arritmia detectada, en los últimos 10 segundos del registro.

Del total de registros de la base de datos se seleccionaron 155 que corresponden a eventos positivos de asístole, bradicardia y taquicardia que a su vez incluyen la FPG. Los 155 registros se distribuyen según: 19 de asístole, 38 de bradicardia y 98 de taquicardia. El algoritmo se realizó en Python 3.4 usando NumPy y SciPy.

\section{Resultados}

La evaluación del algoritmo de detección de picos se muestra en las Tablas 1 y 2.

Tabla 1.

Índices de rendimiento del algoritmo para los registros del 1 al 21. Elaboración propia.

\begin{tabular}{|c|c|c|c|c|c|c|c|c|c|c|}
\hline No. & Señales & $\underset{(\mathbf{m s})}{\mu}$ & $\begin{array}{c}\sigma \\
(\mathbf{m s})\end{array}$ & FP & FN & VP & $\begin{array}{c}\mathrm{Se} \\
(\%)\end{array}$ & $\begin{array}{c}+P \\
(\%)\end{array}$ & $\begin{array}{c}\text { FC } \\
(\mathbf{l p m})\end{array}$ & $\begin{array}{l}\text { FC }_{\text {ref }} \\
\text { (lpm) }\end{array}$ \\
\hline 1 & 0009_8min & 0 & 2 & 0 & 0 & 816 & 100 & 100 & 102 & 102 \\
\hline 2 & 0015_8min & 1 & 2 & 0 & 0 & 960 & 100 & 100 & 120 & 120 \\
\hline 3 & 0016_8min & 2 & 2 & 9 & 0 & 1002 & 100 & 98 & 125 & 126 \\
\hline 4 & 0018_8min & 2 & 2 & 3 & 0 & 1126 & 100 & 99 & 140 & 141 \\
\hline 5 & 0023_8min & 1 & 2 & 0 & 0 & 817 & 100 & 100 & 102 & 102 \\
\hline 6 & 0028_8min & 1 & 2 & 0 & 0 & 588 & 100 & 100 & 73 & 73 \\
\hline 7 & 0029_8min & 3 & 3 & 0 & 0 & 545 & 100 & 100 & 68 & 68 \\
\hline 8 & 0030_8min & -2 & 4 & 2 & 3 & 927 & 99 & 99 & 115 & 117 \\
\hline 9 & 0031_8min & 0 & 2 & 0 & 65 & 546 & 86 & 100 & 67 & 67 \\
\hline 10 & 0032_8min & 1 & 5 & 28 & 2 & 683 & 99 & 95 & 87 & 85 \\
\hline 11 & 0035_8min & 2 & 3 & 18 & 0 & 899 & 100 & 97 & 112 & 112 \\
\hline 12 & 0038_8min & 2 & 4 & 0 & 0 & 956 & 100 & 100 & 119 & 120 \\
\hline 13 & 0103_8min & -1 & 1 & 0 & 0 & 827 & 100 & 100 & 103 & 103 \\
\hline 14 & 0104_8min & 0 & 2 & 0 & 0 & 911 & 100 & 100 & 114 & 114 \\
\hline 15 & 0105_8min & 0 & 2 & 4 & 3 & 527 & 99 & 99 & 65 & 66 \\
\hline 16 & 0115_8min & 1 & 2 & 22 & 0 & 750 & 100 & 96 & 94 & 96 \\
\hline 17 & 0121_8min & 1 & 2 & 0 & 0 & 580 & 100 & 100 & 72 & 72 \\
\hline 18 & 0122_8min & 0 & 2 & 0 & 0 & 588 & 100 & 100 & 72 & 73 \\
\hline 19 & $0123 \_8 \mathrm{~min}$ & -1 & 2 & 6 & 0 & 715 & 100 & 99 & 89 & 90 \\
\hline 20 & 0125_8min & 0 & 2 & 0 & 0 & 626 & 100 & 100 & 78 & 78 \\
\hline 21 & 0127_8min & 0 & 2 & 4 & 0 & 616 & 100 & 99 & 77 & 77 \\
\hline
\end{tabular}




\section{ORANGE JOURNAL}

Tabla 2.

Índices de rendimiento del algoritmo para los registros del 22 al 42. Elaboración propia.

\begin{tabular}{|c|c|c|c|c|c|c|c|c|c|c|}
\hline No. & Señales & $\underset{(\mathbf{m s})}{\boldsymbol{\mu}}$ & $\begin{array}{c}\sigma \\
(\mathbf{m s})\end{array}$ & FP & FN & $\mathbf{V P}$ & $\begin{array}{c}\text { Se } \\
(\%)\end{array}$ & $\begin{array}{c}+P \\
(\%)\end{array}$ & $\begin{array}{c}\text { FC } \\
(\operatorname{lmp})\end{array}$ & $\begin{array}{l}\text { FC } C_{\text {ref }} \\
\text { (lpm) }\end{array}$ \\
\hline 22 & 0128_8min & 1 & 2 & 0 & 0 & 540 & 100 & 100 & 67 & 67 \\
\hline 23 & 0133_8min & -3 & 2 & 0 & 1 & 569 & 99 & 100 & 71 & 71 \\
\hline 24 & 0134_8min & 0 & 2 & 0 & 1 & 578 & 99 & 100 & 72 & 72 \\
\hline 25 & 0142_8min & 0 & 2 & 1 & 0 & 739 & 100 & 99 & 92 & 92 \\
\hline 26 & 0147_8min & 0 & 2 & 8 & 1 & 519 & 100 & 98 & 65 & 66 \\
\hline 27 & 0148_8min & -4 & 2 & 0 & 0 & 623 & 100 & 100 & 78 & 78 \\
\hline 28 & 0149_8min & 1 & 2 & 2 & 0 & 458 & 99 & 100 & 57 & 57 \\
\hline 29 & 0150_8min & 1 & 2 & 12 & 0 & 511 & 100 & 97 & 64 & 64 \\
\hline 30 & 0309_8min & 1 & 1 & 3 & 0 & 510 & 100 & 99 & 63 & 64 \\
\hline 31 & 0311_8min & -4 & 1 & 0 & 0 & 555 & 100 & 100 & 69 & 69 \\
\hline 32 & 0312_8min & 0 & 1 & 23 & 0 & 422 & 100 & 94 & 53 & 54 \\
\hline 33 & 0313_8min & 1 & 1 & 0 & 0 & 587 & 100 & 100 & 73 & 73 \\
\hline 34 & 0322_8min & -6 & 2 & 0 & 0 & 589 & 100 & 100 & 73 & 73 \\
\hline 35 & 0325_8min & 0 & 1 & 0 & 0 & 585 & 100 & 100 & 73 & 73 \\
\hline 36 & 0328_8min & 0 & 1 & 8 & 0 & 636 & 100 & 98 & 79 & 79 \\
\hline 37 & 0329_8min & 1 & 1 & 0 & 0 & 701 & 100 & 100 & 87 & 89 \\
\hline 38 & 0330_8min & 0 & 1 & 0 & 0 & 600 & 100 & 100 & 75 & 75 \\
\hline 39 & 0331_8min & 0 & 1 & 0 & 0 & 538 & 100 & 100 & 67 & 68 \\
\hline 40 & 0332_8min & 1 & 1 & 0 & 0 & 590 & 100 & 100 & 73 & 74 \\
\hline 41 & 0333_8min & 0 & 1 & 8 & 0 & 560 & 100 & 98 & 70 & 72 \\
\hline 42 & 0370_8min & 1 & 1 & 26 & 0 & 577 & 100 & 95 & 71 & 73 \\
\hline & & \multicolumn{2}{|c|}{$0 \pm 2 \mathrm{~ms}$} & 187 & 276 & 27992 & 99 & 99 & & $\pm 1 \mathrm{lpm}$ \\
\hline
\end{tabular}

La figura 2 muestra el proceso de detección paso a paso. Un pico se considera detectado (verdadero positivo) si la salida del algoritmo se encuentra en una ventana de \pm 50 ms alrededor de la anotación.

A continuación, se clasifican 155 registros de la base de datos del PhysioNet/Computing in Cardiology Challenge del 2015. Estos registros contienen eventos de arritmias clasificadas en: Asístole, Bradicardia y Taquicardia. El criterio de clasificación es el siguiente: se define como asístole a la ausencia de ritmo cardiaco por al menos 4 segundos, taquicardia se asocia a una FC por encima de $100 \mathrm{l} / \mathrm{m}$ y la bradicardia a una FC por debajo de $60 \mathrm{lpm}$. En el caso de que el valor de la FC calculada por el algoritmo resulte en el intervalo 60-100 lpm se considera una clase adicional: Normal. No obstante, todos los registros empleados pertenecen a alguna de las tres clases señaladas anteriormente. Cuando la salida del algoritmo resulte Normal, esta se tomará como un FN en el cálculo de la sensibilidad y el valor predictivo positivo. En la Tabla 3 se muestra un resumen de los resultados del algoritmo.

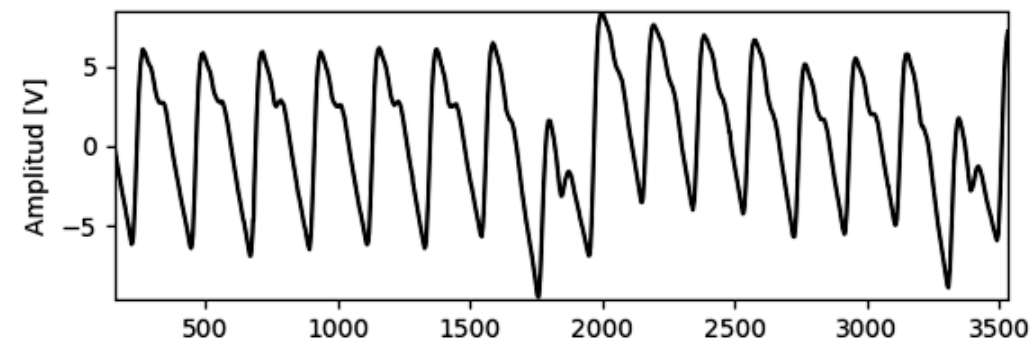




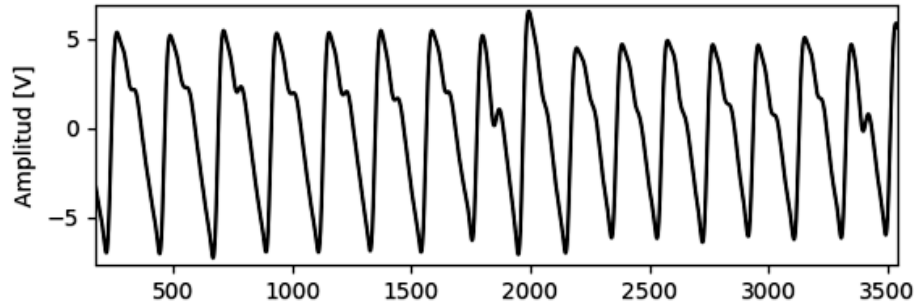

b)

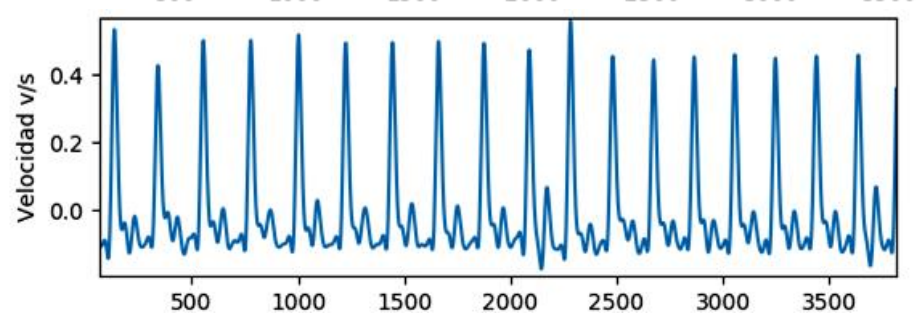

c)

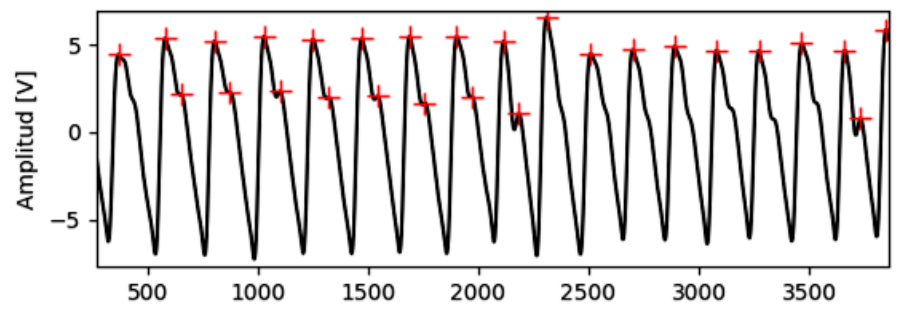

d)

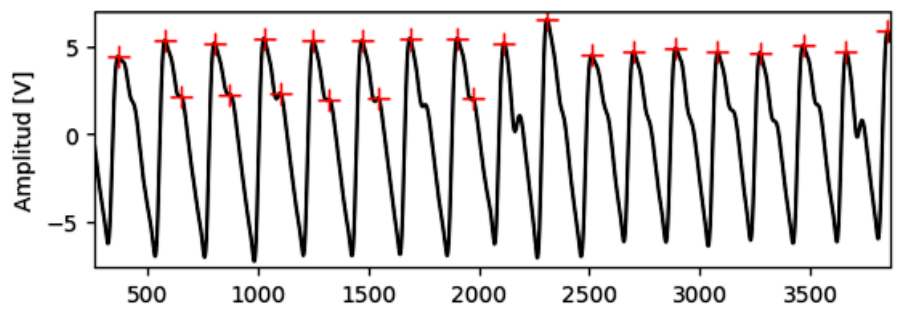

e)

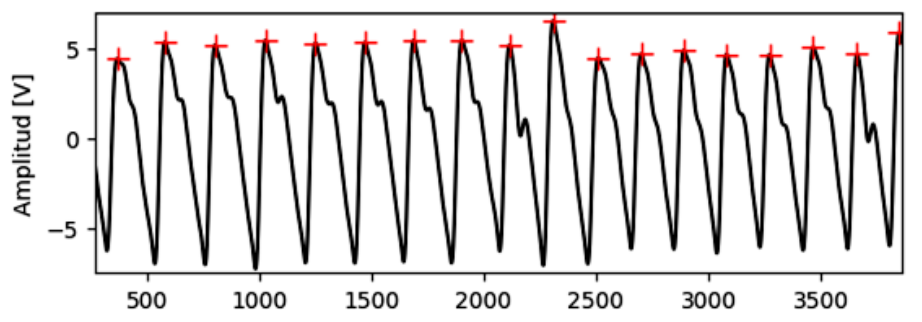

Figura 2. Pasos del algoritmo: a) señal sin filtrar, b) señal filtrada, c) primera derivada, d) selección de candidatos a picos sistólicos según la derivada, e) primera depuración de los candidatos con el umbral adaptativo, f) salida del algoritmo DBSCAN. Elaboración propia.

Tabla 3.

Resultados de la salida del algoritmo en la clasificación de arritmias. Elaboración propia.

\begin{tabular}{|c|c|c|c|c|c|c|c|c|}
\hline \multirow[t]{2}{*}{ Clases } & \multirow{2}{*}{$\begin{array}{l}\text { Cantida } \\
\text { d de } \\
\text { registros }\end{array}$} & \multicolumn{4}{|c|}{ Salida de clasificación } & \multirow{2}{*}{$\begin{array}{l}\text { Se } \\
\%\end{array}$} & \multirow{2}{*}{$\begin{array}{l}\mathbf{P +} \\
\%\end{array}$} & \multirow{2}{*}{$\begin{array}{l}\text { Sp } \\
\%\end{array}$} \\
\hline & & Asístole & Bradicardia & Taquicardia & Normal & & & \\
\hline Asístole & 19 & 15 & 3 & 0 & 1 & 79 & 83 & 96 \\
\hline Bradicardia & 38 & 1 & 27 & 6 & 4 & 71 & 69 & 89 \\
\hline Taquicardia & 98 & 2 & 9 & 85 & 2 & 87 & 93 & 84 \\
\hline Total & 155 & 18 & 39 & 91 & 15 & & & \\
\hline
\end{tabular}




\section{ORANGE JOURNAL}

\section{Discusión}

Los picos no detectados (FN) se deben al umbral seleccionado en el algoritmo (30\% de la amplitud máxima de la señal en la ventana de análisis). Este umbral afecta en mayor medida a las señales que poseen grandes diferencias de amplitud de picos contiguos.

Los falsos positivos (FP) en su mayoría se deben a detecciones donde la morfología de la señal se ve afectada por artefactos. En estas zonas los expertos no realizaron anotaciones en los latidos a ambos lados de la región afectada por no considerarlos latidos completos. En la figura 3 se muestra la detección de dos falsos positivos por este motivo. En el registro número 10 además de presentarse esta situación, los falsos positivos detectados están dados porque el algoritmo detectó 13 picos diastólicos.

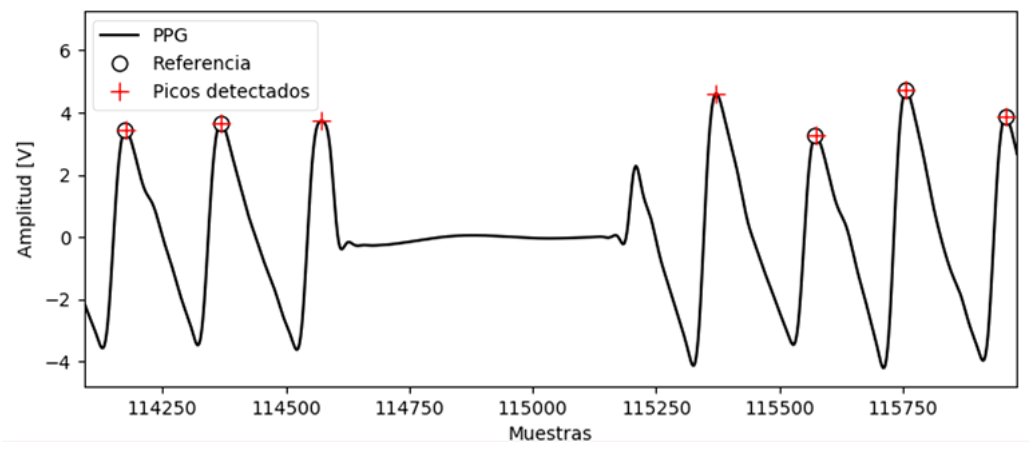

Figura 3. Falsos Positivos detectados por el algoritmo. Elaboración propia.

El algoritmo para la detección de los picos sistólicos de la FPG mostró una a exactitud y precisión altas (0 $\pm 2 \mathrm{~ms})$ al igual que la estimación de la frecuencia cardiaca $(0 \pm 1 \mathrm{lpm})$.

En la detección de arritmias el algoritmo mostró una Se y P+ aceptables, fundamentalmente en la clasificación de taquicardia. Para 5 registros (b311, b124s, b4551, b456s, b5116s), el algoritmo detecta picos diastólicos, lo cual afecta la estimación de la frecuencia cardiaca, dando lugar a una clasificación normal o taquicardia en lugar de bradicardia, ver figura 4a. En 6 registros (t506s, t6791, t7471, t4771, t3431, t7471) con apreciables variaciones de amplitud en el segmento de análisis, el algoritmo no detecta todos los picos existentes, esto trae consigo que la FC estimada disminuya y se detecte como bradicardia o normal un registro con anotación de taquicardia, figura $4 \mathrm{~b}$.

a)

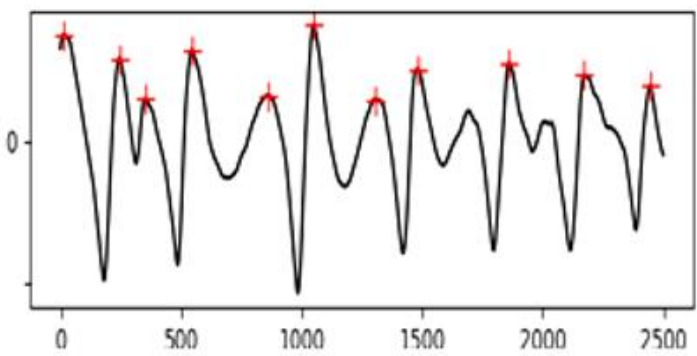

b)

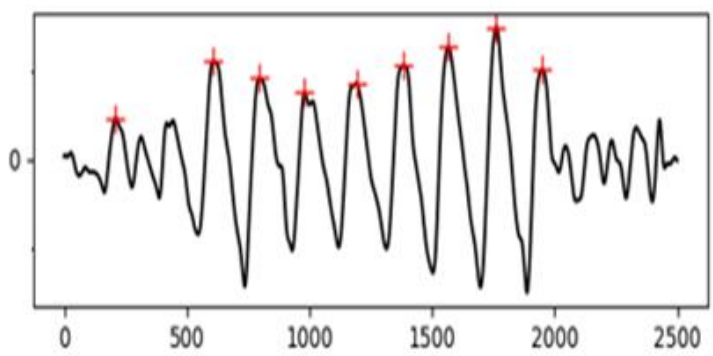

Figura 4. a) Registro b124s con anotación "bradicardia" y salida del algoritmo "normal" debido a la detección de picos diastólicos, b) registro 17471 con anotación "taquicardia" y salida del algoritmo "bradicardia". Elaboración propia.

A esto se suman 18 registros con baja relación señal-ruido que afectan los resultados del algoritmo, por ejemplo el registro a1611, ver figura 5. 


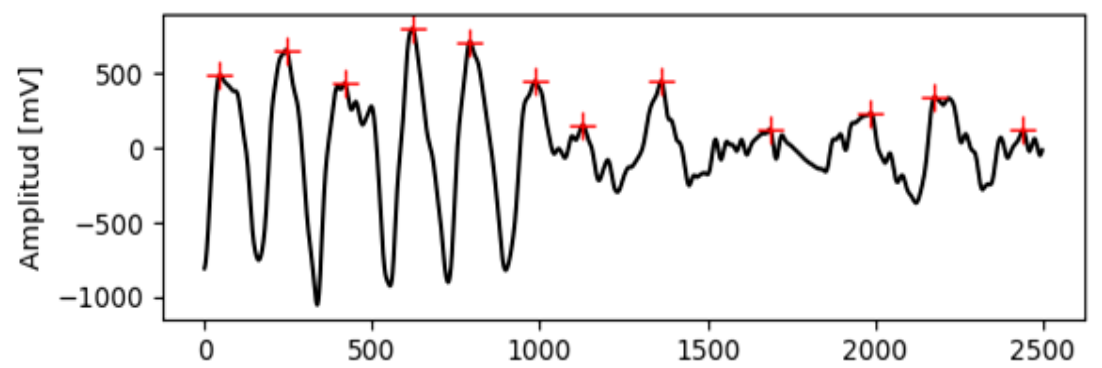

Figura 5. Registro a1611, señal ruidosa con anotación "asístole" y salida del algoritmo "bradicardia". Elaboración propia.

Estos errores son consecuencia de que el umbral calculado se basa en la amplitud máxima de la señal en la ventana de análisis. Esto implica que ante grandes variaciones de amplitud el umbral estimado adquiere un valor elevado provocando la pérdida de picos en la ventana. Además, el método propuesto detecta los picos diastólicos más pronunciados que superan el umbral. Para la primera base de datos la estimación de la FC no se ve afectada por estos fallos del algoritmo ya que la FC estimada se realiza sobre todo el registro. La cantidad de picos existentes en estos registros tienen una media de 666 anotaciones, por lo que pocos FP o FN tienen un menor impacto. Sin embargo, estos fallos tienden a afectar significativamente la estimación de la frecuencia cardiaca para cortos espacios de tiempo. Esto se hace notar más en la segunda base de datos porque el análisis se realiza en 10 segundos de la señal. En esas condiciones un FP o un FN afecta el cálculo de la FC considerablemente.

Para evaluar el rendimiento general del algoritmo se calculó la exactitud (Acc) y el error (Err) global definidos según,

$$
A c c=\frac{\sum_{i=1}^{3} V P_{i}}{\sum_{i=1}^{3} V P_{i}+V N_{i}+F P_{i}+F N_{i}} \quad E r r=\frac{\sum_{i=1}^{3} F P_{i}+F N_{i}}{\sum_{i=1}^{3} V P_{i}+V N_{i}+F P_{i}+F N_{i}}
$$

Donde: $V P$-Verdadero Positivo, $V N$-Verdadero Negativo, $F N$-Falso Negativo, FP-Falso Positivo

El algoritmo tiene una exactitud global de un $82 \%$ y un error del $18 \%$. Aunque el algoritmo clasifica correctamente las señales menos ruidosas, la frecuencia cardiaca en intervalos cortos de tiempo en señales con baja relación señal-ruido o contaminadas con artefactos es un indicador poco robusto. En consecuencia, la detección de arritmias por este método se ve comprometida sustancialmente.

En (Paradkar \& Chowdhury, 2017) se propone un método para detectar la presencia de 5 clases de arritmias utilizando la señal de FPG:: taquicardia, bradicardia, taquicardia ventricular y fibrilación ventricular. Este estudio utiliza también la base de datos del PhysioNet/Computing in Cardiology Challenge del 2015. Los autores utilizan un total de 569 señales ya que su objetivo principal es disminuir falsas alarmas en la detección de arritmias por lo que utilizan registros con presencia o no de una de las 5 clases. En la Tabla 4 se muestran los valores de Se y Sp para ambos estudios.

Tabla 4.

Tabla comparativa entre dos métodos de detección de arritmias por FPG. Elaboración propia.

\begin{tabular}{|c|c|c|c|c|c|c|}
\hline \multirow{3}{*}{ Estudios } & \multicolumn{6}{|c|}{ Tipos de arritmia } \\
\hline & \multicolumn{2}{|c|}{ Asístole } & \multicolumn{2}{|c|}{ Bradicardia } & \multicolumn{2}{|c|}{ Taquicardia } \\
\hline & $\operatorname{Se}(\%)$ & $\mathrm{Sp}(\%)$ & Se $(\%)$ & $\mathrm{Sp}(\%)$ & $\operatorname{Se}(\%)$ & $\mathrm{Sp}(\%)$ \\
\hline $\begin{array}{ll}\text { (Paradkar } & \& \\
\text { Chowdhury, 2017) } & \end{array}$ & 85 & 95 & 88 & 73 & 97 & 77 \\
\hline Este estudio & 79 & 96 & 71 & 89 & 87 & 84 \\
\hline
\end{tabular}


El presente estudio muestra una Se más baja, sin embargo, posee una Sp más elevada. Aunque en (Paradkar \& Chowdhury, 2017) no se brindan los valores de P+ y Acc, observando el comportamiento de la Se y Sp se puede deducir que el método que se propone en este estudio tiende a detectar menos FP. Además se debe agregar que en (Paradkar \& Chowdhury, 2017) se realiza una evaluación de la calidad de la señal. Los registros utilizados son aquellos que cumplen al menos con el $60 \%$ del índice de calidad de la señal calculado.

\section{Conclusiones}

El algoritmo de detección de picos sistólicos propuesto muestra una alta exactitud y precisión con la primera base de datos, no obstante falla ante señales con grandes diferencias de amplitud entre picos relativamente cercanos. La clasificación de arritmias basadas en la utilización de la frecuencia cardiaca extraída de la señal de fotopletismografía es posible en señales relativamente limpias y con pocos artefactos. Aunque la efectividad del método propuesto puede afectarse en registros de señales con grandes variaciones de amplitud o con relaciones señal-ruido bajas, los resultados en estas condiciones son aceptables y muy buenos en señales de calidad. En circunstancias de bajo SNR se pueden evaluar métodos avanzados de preprocesamiento u otros enfoques como: inteligencia artificial, modelos gaussianos, máquinas de vectores soporte, entre otros. Estos métodos no solo se basan en la estimación de la frecuencia cardiaca para la clasificación de arritmias, sino que explotan información de otras características de la señal.

\section{Referencias Bibliográficas}

Allen, J. (2007). Photoplethysmography and its application in clinical physiological measurement. Physiological measurement, 28(3), R1.

Asensi, J. O., de Francisco, M. T. I., Pérez, Ó. C., de Carranza, M. J. S. T., Rubio, J. A., Palop, C. P., ... \& Dolz, L. M. (2021). Estudio RITHMI, capacidad diagnóstica de un monitor de ritmo cardiaco para la detección automática de fibrilación auricular. Revista Española de Cardiología, 74(7), 602-607.

Bonomi, A. G., Schipper, F., Eerikäinen, L. M., Margarito, J., Van Dinther, R., Muesch, G., ... \& Dekker, L. R. (2018). Atrial fibrillation detection using a novel cardiac ambulatory monitor based on photo-plethysmography at the wrist. Journal of the American Heart Association, 7(15), e009351.

Castaneda, D., Esparza, A., Ghamari, M., Soltanpur, C., \& Nazeran, H. (2018). A review on wearable photoplethysmography sensors and their potential future applications in health care. International Journal of Biosensors \& Bioelectronics, 4, 195-202. doi: DOI: 10.15406/ijbsbe.2018.04.00125

Clifford, G. D., Silva, I., Moody, B., Li, Q., Kella, D., Shahin, A., ... \& Mark, R. G. (2015). The PhysioNet/computing in cardiology challenge 2015: reducing false arrhythmia alarms in the ICU. Paper presented at the 2015 Computing in Cardiology Conference (CinC).

Constantén, S.B., Pepe, D.S and Mona, P. (2020). Anuario Estadístico de Salud 2020. Ministerio de Salud Pública de Cuba.

Coviello, J. S. (2020). ECG interpretation made incredibly easy!: Lippincott Williams \& Wilkins.

Eerikäinen, L. M., Vanschoren, J., Rooijakkers, M. J., Vullings, R., \& Aarts, R. M. (2015, September). Decreasing the false alarm rate of arrhythmias in intensive care using a machine learning approach. In 2015 Computing in Cardiology Conference (CinC) (pp. 293-296). IEEE.

Ester, M., Kriegel, H. P., Sander, J., \& Xu, X. (1996, August). A density-based algorithm for discovering clusters in large spatial databases with noise. In kdd (Vol. 96, No. 34, pp. 226-231).

Fallet, S., Yazdani, S., \& Vesin, J. M. (2015, September). A multimodal approach to reduce false arrhythmia alarms in the intensive care unit. In 2015 Computing in Cardiology Conference (CinC) (pp. 277280). IEEE.

García, E. G., Gómez, F. G., Pion, M. G., \& Alonso-Colmenares, M. G. (2015). Arritmias cardíacas en urgencias. Medicine-Programa de Formación Médica Continuada Acreditado, 11(87), 5175-5184.

Guyton, A.C. (2006). Tratado de fisiologia medica. Brasil: Elsevier

Jung, D. K., Kim, G. R., Kim, K. N., Choi, B. C., Suh, D. J., Jeon, G. R., \& Ye, S. Y. (2007). Changes of pulse wave velocity in arm according to characteristic points of pulse wave. Paper presented at the 2007 International Conference on Convergence Information Technology (ICCIT 2007). 
Kalidas, V. and Tamil, L. S. (2015). Enhancing accuracy of arrhythmia classification by combining logical and machine learning techniques. Paper presented at the 2015 Computing in Cardiology Conference (CinC).

Karlen, W., Raman, S., Ansermino, J. M., \& Dumont, G. A. (2013). Multiparameter respiratory rate estimation from the photoplethysmogram. IEEE Transactions on Biomedical Engineering, 60(7), 1946-1953.

Paradkar, N., \& Chowdhury, S. R. (2017). Cardiac arrhythmia detection using photoplethysmography. Paper presented at the 2017 39th Annual International Conference of the IEEE Engineering in Medicine and Biology Society (EMBC).

Poh, M. Z., Poh, Y. C., Chan, P. H., Wong, C. K., Pun, L., Leung, W. W. C., ... \& Siu, C. W. (2018). Diagnostic assessment of a deep learning system for detecting atrial fibrillation in pulse waveforms. Heart, 104(23), 1921-1928.

Polanía, L. F., Mestha, L. K., Huang, D. T., \& Couderc, J. P. (2015). Method for classifying cardiac arrhythmias using photoplethysmography. Paper presented at the 2015 37th Annual International Conference of the IEEE Engineering in Medicine and Biology Society (EMBC).

Rezaei Yousefi, Z. (2018). Atrial Fibrillation Detection from Photoplethysmography Data Using Artificial Neural Networks. (Master), Tampere University of Tecnology.

Sadr, N., Huvanandana, J., Nguyen, D. T., Kalra, C., McEwan, A., \& de Chazal, P. (2015, September). Reducing false arrhythmia alarms in the ICU by Hilbert QRS detection. In 2015 Computing in Cardiology Conference (CinC) (pp. 1173-1176). IEEE.

Saquib, N., Papon, M. T. I., Ahmad, I., \& Rahman, A. (2015). Measurement of heart rate using photoplethysmography. In 2015 International Conference on Networking Systems and Security (NSysS) (pp. 1-6). IEEE.

Shashikumar, S. P., Shah, A. J., Li, Q., Clifford, G. D., \& Nemati, S. (2017, February). A deep learning approach to monitoring and detecting atrial fibrillation using wearable technology. In 2017 IEEE EMBS International Conference on Biomedical \& Health Informatics (BHI) (pp. 141-144). IEEE.

Stankevičius, D., Petrènas, A., Sološenko, A., Grigutis, M., Januškevičius, T., Rimševičius, L., \& Marozas, V. (2016). Photoplethysmography-based system for atrial fibrillation detection during hemodialysis. In XIV Mediterranean Conference on Medical and Biological Engineering and Computing 2016 (pp. 79-82). Springer, Cham.

Vázquez Seisdedos, C.R. (2001). Análisis del ECG en monitorización ambulatoria para el diagnóstico cardíaco y la predicción de muerte cardíaca súbita. Universdtat Politécnica de Catalunya. 